\title{
Black hole radiance, short distances, and TeV gravity
}

\author{
Iván Agulló, José Navarro-Sala: 田 \\ Departamento de Física Teórica and IFIC, Centro Mixto Universidad de Valencia-CSIC \\ Universidad de Valencia, Burjassot-46100, Valencia, Spain \\ Gonzalo J. Olmd \\ Physics Department, University of Wisconsin-Milwaukee, P.O. Box 413, Milwaukee, Wisconsin 53201 USA
}

(Dated: April 6, 2006)

\begin{abstract}
Using a derivation of black hole radiance in terms of two-point functions one can provide a quantitative estimate of the contribution of short distances to the spectrum. Thermality is preserved for black holes with $\kappa l_{P}<<1$. However, deviations from the Planckian spectrum can be found for mini black holes in $\mathrm{TeV}$ gravity scenarios, even before reaching the Planck phase.
\end{abstract}

PACS numbers: 04.70.Dy, 04.50.+h, 11.10Kk

Black hole radiance is one of the most important consequences of combining general relativity and quantum mechanics. Using quantum field theory in curved spacetime Hawking [1] showed that a black hole emits thermal radiation. The derivation involves considering arbitrarily high frequency wave-packets in the intermediate states of the derivation. Any out-going Hawking quanta with finite energy at infinity will have an exponentially increasing frequency when it is propagated backwards in time and measured by a free-falling observer at the horizon. The crucial role played by these ultrahigh frequencies in the derivation of the Planckian spectrum, or equivalently, the short-distance behavior of the free field considered, was stressed in [2, 3]. This question has been mainly analyzed using sonic black hole models with modified high frequency dispersion relations [4, 5] so as to eliminate ultrashort wavelength modes. In doing so one must assume the existence of a preferred frame. Such a frame is naturally identified with the rest frame of the atoms of the fluid and the modified dispersion relations come from effects of its microscopic structure. The result is that, even with a drastic change of the theory, thermality is essentially unaffected if the black hole scale is far from the underlying microscopic scale. This does not exclude that, for small black holes, with size not too far from the fundamental length scale, the standard Planckian spectrum can be modified.

The purpose of this paper is to analyze this issue, in a purely gravitational context, in terms of two-point functions instead of dispersion relations. This way the shortdistance contribution to the spectrum can be evaluated in a more explicit way. We focus our analysis on the situation where non-trivial deviations from thermality can be found, even before reaching the late stages (Planck scale) of the evaporation. Therefore we shall pay particular attention to mini black holes considered recently $[\underline{6}, 7,[8]$ in $\mathrm{TeV}$ gravity scenarios. The existence of extra dimensions

\footnotetext{
*Electronic address: ivan.agullo@uv.es; jnavarro@ific.uv.es
}

${ }^{\dagger}$ Electronic address: olmoalba@uwm.edu gives hope to the possibility that the fundamental Planck mass could be TeV order [9]. This, in turn, opens the viability of producing black holes by high energy collisions 10] (as in the LHC or in cosmic ray scattering) and detecting the Standard Model quanta of Hawking radiation 11. Such black holes need to be very small (less than the typical length of extra dimensions) and above the fundamental Planck scale to apply semiclassical gravity. In this scenario measurable deviations from thermality can arise due to unknown physics at ultrashort distances.

The mean particle number produced in the gravitational collapse of a rotating black hole is

$$
\left\langle N_{i}\right\rangle=\frac{\Gamma_{i}}{e^{2 \pi \kappa^{-1}\left(w_{i}-m \Omega_{H}\right)}-(-)^{2 s}},
$$

where $\kappa$ and $\Omega_{H}$ are the surface gravity and the angular velocity, respectively, of the black hole horizon. The $\Gamma_{i}$ are grey-body factors, associated to a wave-packet $i$-mode (sharply peaked around the frequency $\omega_{i}$ ) of a given particle species of spin $s$, and $m$ is the axial angular momentum of the emitted particle. Up to grey-body coefficients the spectrum is purely Planckian with the chemical potential term $m \Omega_{H}$. Note that the scale of (11) is essentially given by the (classical) surface gravity $\kappa$ of the black hole. Moreover the radiation is exactly thermal in the sense that there is no correlation between different modes $(i \neq j)$

$$
\left\langle N_{i} N_{j}\right\rangle=\left\langle N_{i}\right\rangle\left\langle N_{j}\right\rangle
$$

When the modes coincide $(i=j)$ the result is consistent with the thermal probability distribution and the state of radiation is indeed described by a thermal density matrix [12, 13] (see also [14, 15]).

The above results are consequence of the evaluation of the late-time Bogolubov coefficients in a gravitational collapse. The expansion of a field in two different sets of positive frequency modes: $u_{j}^{i n}(x)$ (in the past infinity) and $u_{j}^{\text {out }}(x)$ (in the future infinity) leads to a relation for the corresponding creation and annihilation operators: $a_{i}^{\text {out }}=\sum_{j}\left(\alpha_{i j}^{*} a_{j}^{\text {in }}-\beta_{i j}^{*} a_{j}^{i n \dagger}\right)$. When the coefficients $\beta_{i j}$ do not vanish the vacuum states $|i n\rangle$ and $\mid$ out $\rangle$ 
do not coincide and, therefore, the number of particles measured in the $i^{t h}$ mode by an "out" observer, in the state $|i n\rangle$ is given by $\left\langle i n\left|N_{i}^{\text {out }}\right| i n\right\rangle=\sum_{k}\left|\beta_{i k}\right|^{2}$. Moreover the correlations for $i \neq j$ are given by $\left\langle i n\left|N_{i} N_{j}\right| i n\right\rangle=$ $\left(\sum_{k}\left|\beta_{i k}\right|^{2}\right)\left(\sum_{k}\left|\beta_{j k}\right|^{2}\right)+\left|\sum_{k} \beta_{i k} \beta_{j k}^{*}\right|^{2}+\left|\sum_{k} \alpha_{i k} \beta_{j k}\right|^{2}$. The use of the above relations and the explicit evaluation of the matrices $\beta_{i j}$ and $\alpha_{i j}$ at late-times, which always involves to consider intermediate ultrahigh frequency modes (due to the exponential redshift associated to the black hole horizon), leads to the thermal results (11) and (2).

Within the standard analysis in terms of Bogolubov coefficients it is not easy to evaluate explicitly how ultrahigh frequencies or, equivalently, ultrashort distances contribute to generate the thermal spectrum. However, it is not difficult to rederive the Hawking effect in such a way that the contribution of short-distance physics can be explicitly worked out. Let us assume, for the sake of simplicity, that $\phi$ is a massless, neutral and minimally coupled scalar field. One can easily verify that the number operator can be obtained from the following projection

$$
\begin{aligned}
a_{i}^{\text {out }}{ }_{i}^{\dagger} a_{j}^{\text {out }}= & \int_{\Sigma} d \Sigma_{1}^{\mu} d \Sigma_{2}^{\nu}\left[u_{i}^{\text {out }}\left(x_{1}\right) \stackrel{\leftrightarrow}{\partial}_{\mu}\right]\left[u_{j}^{\text {out } *}\left(x_{2}\right) \overleftrightarrow{\partial}_{\nu}\right] \\
& \times\left(\phi\left(x_{1}\right) \phi\left(x_{2}\right)-\left\langle\text { out }\left|\phi\left(x_{1}\right) \phi\left(x_{2}\right)\right| \text { out }\right\rangle\right)
\end{aligned}
$$

where $\Sigma$ represents a suitable initial value hypersurface and the two-point expectation value has the form $\left\langle\right.$ out $\left|\phi\left(x_{1}\right) \phi\left(x_{2}\right)\right|$ out $\rangle=\hbar \sum_{k} u_{k}^{\text {out }}\left(x_{1}\right) u_{k}^{\text {out }}{ }^{*}\left(x_{2}\right)$. Therefore, the number of particles in the $i^{\text {th }}$ mode measured by the "out" observer in the "in" vacuum is given by $\left\langle i n\left|N_{i}\right| i n\right\rangle \equiv\left\langle i n\left|N_{i i}\right| i n\right\rangle$, where $N_{i j} \equiv \hbar^{-1} a^{\text {out }}{ }_{i}^{\dagger} a_{j}^{\text {out }}$, and it can be evaluated using the above expression. In twodimensions analogous formulae have been worked out in 16 and a somewhat related scheme has been given in [17.

Let us now apply (3) to the formation process of a Schwarzschild black hole and restrict the "out" region to future null infinity $\left(I^{+}\right)$. The "in" region is, as usual, defined by past null infinity $\left(I^{-}\right)$. At $I^{+}$we can consider the normalized radial plane-wave modes $u_{w l m}^{\text {out }}(t, r, \theta, \phi)=$ $u_{w}(u) r^{-1} Y_{l m}(\theta, \phi)$, where $u_{w}(u)=\frac{e^{-i w u}}{\sqrt{4 \pi w}}$ and $u$ is the null retarded time. Note that to work with the null hypersurface $I^{+}$instead of a spacelike one requires to replace the two-point function by the symmetrized one. We shall now evaluate the matrix coefficients $\left\langle i n\left|N_{i_{1} i_{2}}\right| i n\right\rangle$ where $i \equiv(w, l, m)$. After straightforward manipulations we have

$$
\begin{gathered}
\left\langle i n\left|N_{i_{1} i_{2}}\right| i n\right\rangle=\frac{4}{\hbar} \int_{I^{+}} d u_{1} d \Omega_{1} d u_{2} d \Omega_{2} Y_{l_{1} m_{1}}\left(\theta_{1}, \phi_{1}\right) \times \\
Y_{l_{2} m_{2}}^{*}\left(\theta_{2}, \phi_{2}\right) u_{w_{1}}\left(u_{1}\right) u_{w_{2}}^{*}\left(u_{2}\right) \partial_{u_{1}} \partial_{u_{2}}\left[G_{i n}\left(x_{1}, x_{2}\right)\right. \\
\left.-G_{\text {out }}\left(x_{1}, x_{2}\right)\right],
\end{gathered}
$$

where $G_{i n}\left(x_{1}, x_{2}\right)$ and $G_{\text {out }}\left(x_{1}, x_{2}\right)$ are the two-point functions of the "in" and "out" states, respectively. Note that $G_{\text {in }}\left(x_{1}, x_{2}\right)-G_{\text {out }}\left(x_{1}, x_{2}\right)$ is a smooth function. The singularity of $G_{i n}\left(x_{1}, x_{2}\right)$ is exactly cancelled by the corresponding one of $G_{\text {out }}\left(x_{1}, x_{2}\right)$. At $I^{+}$these functions can be expanded as

$$
\begin{aligned}
G_{\text {out }}\left(x_{1}, x_{2}\right) & =\frac{\hbar}{2} \int_{0}^{\infty} d w \sum_{l, m} \frac{e^{-i w u_{1}}}{\sqrt{4 \pi w}} Y_{l m}\left(\theta_{1}, \phi_{1}\right) \\
& \times \frac{e^{i w u_{2}}}{\sqrt{4 \pi w}} Y_{l m}^{*}\left(\theta_{2}, \phi_{2}\right)+c . c .,
\end{aligned}
$$

and

$$
\begin{aligned}
G_{i n}\left(x_{1}, x_{2}\right) & =\frac{\hbar}{2} \int_{0}^{\infty} d w \sum_{l, m} \frac{e^{-i w v\left(u_{1}\right)}}{\sqrt{4 \pi w}} Y_{l m}\left(\theta_{1}, \phi_{1}\right) \\
& \times \frac{e^{i w v\left(u_{2}\right)}}{\sqrt{4 \pi w}} Y_{l m}^{*}\left(\theta_{2}, \phi_{2}\right)+c . c .
\end{aligned}
$$

where the function $v(u)$ in (6) is, as usual, given by

$$
v \approx \text { constant }-\kappa^{-1} e^{-\kappa u}
$$

Note that this expression, relating the inertial times at $I^{+}$and at $I^{-}$, encodes the effect of the time-dependent gravitational collapse. Using it assumes that we are in the late-time regime and also that we are neglecting the backreaction.

Performing first the angular integrations and defining

$$
\begin{aligned}
\tilde{G}_{\text {out }}\left(u_{1}, u_{2}\right) & \equiv \hbar \partial_{u_{1}} \partial_{u_{2}} \int_{0}^{\infty} d w \frac{e^{-i w\left(u_{1}-u_{2}\right)}}{4 \pi w} \\
& =-\frac{\hbar}{4 \pi} \frac{1}{\left(u_{1}-u_{2}\right)^{2}}
\end{aligned}
$$

and a similar expression for the "in" vacuum

$$
\tilde{G}_{i n}\left(v_{1}, v_{2}\right)=-\frac{\hbar}{4 \pi} \frac{1}{\left(v_{1}-v_{2}\right)^{2}},
$$

we easily get

$$
\begin{gathered}
\left\langle i n\left|N_{i_{1} i_{2}}\right| i n\right\rangle=\frac{\hbar^{-1}}{\pi \sqrt{\omega_{1} \omega_{2}}} \int_{I^{+}} d u_{1} d u_{2} e^{-i\left(w_{1} u_{1}-w_{2} u_{2}\right)} \\
\times\left[\frac{d v_{1}}{d u_{1}} \frac{d v_{2}}{d u_{2}} \tilde{G}_{i n}\left(v_{1}, v_{2}\right)-\tilde{G}_{\text {out }}\left(u_{1}, u_{2}\right)\right] \delta_{l_{1} l_{2}} \delta_{m_{1} m_{2}}(10)
\end{gathered}
$$

We can rewrite this expression using (7) and introducing new variables $z^{+}=u_{2}+u_{1}, z=u_{2}-u_{1}$ so that the integral corresponding to $z^{+}$leads to a delta function in frequencies. The result is

$$
\begin{aligned}
\left\langle i n\left|N_{i_{1} i_{2}}\right| i n\right\rangle & =-\frac{\delta\left(w_{1}-w_{2}\right)}{2 \pi \sqrt{w_{1} w_{2}}} \int_{-\infty}^{+\infty} d z e^{-i \frac{\left(w_{1}+w_{2}\right)}{2} z} \\
& \times\left[\frac{\kappa^{2} e^{-\kappa z}}{\left(e^{-\kappa z}-1\right)^{2}}-\frac{1}{z^{2}}\right] \delta_{l_{1} l_{2}} \delta_{m_{1} m_{2}} \cdot(11)
\end{aligned}
$$

Finally, performing the integration in $z=u_{2}-u_{1}$ we get the Planckian spectrum (see (16)-(17))

$$
\frac{-1}{2 \pi w} \int_{-\infty}^{+\infty} d z e^{-i w z}\left[\frac{\kappa^{2} e^{-\kappa z}}{\left(e^{-\kappa z}-1\right)^{2}}-\frac{1}{z^{2}}\right]=\frac{1}{e^{2 \pi w \kappa^{-1}}-1}(12)
$$


We note that, to obtain this result, we have to assume that quantum field theory is valid on all scales.

To get the final result we have to take into account the fact that we have restricted our "out" Fock space to the external region $I^{+}$. This means that a fraction of an outgoing wave-packet will be scattered by the potential barrier and only part of it reaches $I^{+}$. To incorporate this effect we should multiply the "out" modes $u_{w l m}^{\text {out }}$ in (4) by the transmission coefficients $t_{w l}$ of the Schwarzschild geometry. Therefore we obtain the complete emission rate per unit frequency $w$ and time $u$

$$
\frac{d N}{d w d u} \equiv \frac{1}{2 \pi}\left\langle i n\left|N_{w}\right| i n\right\rangle=\frac{1}{2 \pi} \frac{\Gamma_{l m}}{e^{2 \pi w \kappa^{-1}}-1},
$$

where $\Gamma_{l m}=\left|t_{l m}\right|^{2}$ are the grey-body coefficients. When the black hole is rotating the result is similar to (12) with the replacement of $w$ by $w-m \Omega_{H}$. The analysis can also be extended to account for correlations between number operators with different frequencies. They can be expressed as [18] $\left\langle i n\left|N_{i_{1}} N_{i_{2}}\right| i n\right\rangle-\left\langle i n\left|N_{i_{1}}\right| i n\right\rangle\left\langle i n\left|N_{i_{2}}\right| i n\right\rangle=$ $\left|\left\langle i n\left|N_{i_{1} i_{2}}\right| i n\right\rangle\right|^{2}+\left|\left\langle i n\left|C_{i_{1} i_{2}}\right| i n\right\rangle\right|^{2}$, where $C_{i_{1} i_{2}}$ is the operator

$$
\begin{aligned}
C_{i_{1} i_{2}} & =\int_{\Sigma} d \Sigma_{1}^{\mu} d \Sigma_{2}^{\nu}\left[u_{i_{1}}^{\text {out } *}\left(x_{1}\right) \stackrel{\leftrightarrow}{\partial}_{\mu}\right]\left[u_{i_{2}}^{\text {out } *}\left(x_{2}\right) \stackrel{\leftrightarrow}{\partial}_{\nu}\right] \\
& \times\left(\phi\left(x_{1}\right) \phi\left(x_{2}\right)-\left\langle\text { out }\left|\phi\left(x_{1}\right) \phi\left(x_{2}\right)\right| \text { out }\right\rangle\right)
\end{aligned}
$$

Explicit evaluation gives

$$
\begin{gathered}
\left\langle i n\left|C_{i_{1} i_{2}}\right| i n\right\rangle=-\frac{\delta\left(w_{1}+w_{2}\right)}{2 \pi \sqrt{w_{1} w_{2}}} \int_{-\infty}^{+\infty} d z e^{-i \frac{\left(w_{2}-w_{1}\right)}{2} z} \\
\times\left[\frac{\kappa^{2} e^{-\kappa z}}{\left(e^{-\kappa z}-1\right)^{2}}-\frac{1}{z^{2}}\right] \delta_{l_{1} l_{2}} \delta_{m_{1} m_{2}} .
\end{gathered}
$$

We note that the behavior of the two-point functions (8) and (9) (both $\frac{d v_{1}}{d u_{1}} \frac{d v_{2}}{d u_{2}} \tilde{G}_{i n}\left(v_{1}, v_{2}\right)$ and $\tilde{G}_{\text {out }}\left(u_{1}, u_{2}\right)$ can be expressed in terms of $\left.z=u_{2}-u_{1}\right)$ is fundamental for the vanishing of both quantities $\left\langle i n\left|C_{i_{1} i_{2}}\right| i n\right\rangle$ and $\left\langle i n\left|N_{i_{1} i_{2}}\right| i n\right\rangle$ (the latter with $i_{1} \neq i_{2}$ ).

The expression (12) is very useful since it offers an explicit way to evaluate the "weight" of distances $\left|u_{2}-u_{1}\right|$ to the Planckian spectrum. To be more explicit we shall now compute the contribution of distances $z \in[-\epsilon, \epsilon]$ to the full integral. This contribution

$$
I(w, \kappa, \epsilon)=\frac{-1}{2 \pi w} \int_{-\epsilon}^{+\epsilon} d z e^{-i w z}\left[\frac{\kappa^{2} e^{-\kappa z}}{\left(e^{-\kappa z}-1\right)^{2}}-\frac{1}{z^{2}}\right](16)
$$

can be evaluated analytically

$$
\begin{aligned}
& I(w, \kappa, \epsilon)=1-\frac{1}{2 \pi w}\left\{i w \left[-\frac{2 \kappa}{w} \sin w \epsilon-i \pi-2 i S i(w \epsilon)+\right.\right. \\
& i \frac{\kappa e^{-i w \epsilon}}{w}\left\{{ }_{2} F_{1}\left[1,-\frac{i \omega}{\kappa}, 1-\frac{i \omega}{\kappa}, e^{\kappa \epsilon}\right]\right\}- \\
& \left.i \frac{\kappa e^{i w \epsilon}}{w}\left\{{ }_{2} F_{1}\left[1,-\frac{i w}{\kappa}, 1-\frac{i w}{\kappa}, e^{-\kappa \epsilon}\right]\right\}\right]+\frac{e^{-i \epsilon w}}{\epsilon\left(e^{\epsilon \kappa}-1\right)} \times \\
& \left.\left[-1+e^{\epsilon(\kappa+2 i w)}+e^{\epsilon \kappa}(1-\epsilon \kappa)-e^{2 i \epsilon w}(1+\epsilon \kappa)\right]\right\},
\end{aligned}
$$

and in the limit $\epsilon \rightarrow+\infty$ we nicely recover the Planckian spectrum $\left(e^{2 \pi w \kappa^{-1}}-1\right)^{-1}$. We note that the above expression holds equally for an arbitrary number $4+n$ of dimensions. Moreover, a simple calculation shows that the absence of correlations $\left\langle i n\left|N_{i_{1}} N_{i_{2}}\right| i n\right\rangle-$ $\left\langle i n\left|N_{i_{1}}\right| i n\right\rangle\left\langle i n\left|N_{i_{2}}\right| i n\right\rangle=0$ in the emitted radiation is preserved even if short distances are excluded in the evaluation of $\left\langle i n\left|C_{i_{1} i_{2}}\right| i n\right\rangle$ and $\left\langle i n\left|N_{i_{1} i_{2}}\right| i n\right\rangle$.

For black holes produced by gravitational stellar collapse the contribution of $I(w, \kappa, \epsilon)$ is, when $\epsilon$ is taken as the Planck length $l_{P}=1.6 \times 10^{-33} \mathrm{~cm}$, negligible (of order $\kappa \epsilon$ for $w_{\text {typical }} \sim \kappa / 2 \pi \equiv T_{H}$ ). In fact, for a black hole of three solar masses we need high frequencies $w / w_{\text {typical }} \approx 96$ to find that the contribution of Planck distances $I\left(w, \kappa, l_{P}\right)$ is of order of the total spectrum itself. Moreover, the relative contribution to the Planckian distribution is, for $w=w_{\text {typical }}$, of order $10^{-38 \%}$. For primordial black holes $M \approx 10^{15} \mathrm{~g}$ we find $w / w_{\text {typical }} \approx 52$ and the relative contribution to the spectrum is now $10^{-19 \%}$. This is why Hawking thermal radiation is very robust, as it has been confirmed in analysis based on acoustic black holes (for recent reviews, see [19]). The condition on $\left|u_{1}-u_{2}\right|$, which accounts for very short wavelength, is analogous to the modification of the dispersion relations in the fluid frame. The deviations from the Planckian spectrum are also found, in acoustic black holes, of order $\kappa k_{0}\left(k_{0}\right.$ is the wave vector characterizing the fluid atomic scale) for $w \sim w_{\text {typical }}$.

When the product $\epsilon \kappa$ is of unit order the contribution of short distances to the Planckian spectrum is not negligible. The integral $I(w, \kappa, \epsilon)$ gives values similar to $\left(e^{2 \pi w \kappa^{-1}}-1\right)^{-1}$ when $w / w_{\text {typical }}$ is not very high. This happens in $\mathrm{TeV}$ gravity scenarios. Assuming a drastic change of the strength of gravity at short distances due to $n$ extra dimensions (a Planck mass $M_{T e V}$ of $1 \mathrm{TeV}$ ) and for a $(4+n)$-dimensional Schwarzschild black hole of mass $M(M \sim 5-10 \mathrm{TeV})$ with [20] $\kappa=\frac{(n+1)}{2 r_{H}}$, where the horizon radius is given by

$r_{H}=\frac{2}{M_{T e V}}\left(\frac{M}{M_{T e V}}\right)^{\frac{1}{n+1}}\left(\frac{\pi^{(n-3) / 2} \Gamma((n+3) / 2)}{n+2}\right)^{\frac{1}{n+1}}$, we obtain: $w / w_{\text {typical }} \approx 3.3(n=2), w / w_{\text {typical }} \approx 3.1$

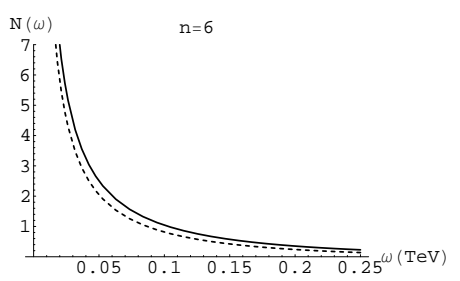

FIG. 1: Plot comparing the Planckian distribution (solid line) $N(w, \kappa)=\left(e^{2 \pi w / \kappa}-1\right)^{-1}$ with the one obtained by suppressing the contributions coming from distances shorter than $\epsilon=l_{T e V}$ (dotted line). We have taken $M=10 \mathrm{TeV}$.

$(n=4)$ and $w / w_{\text {typical }} \approx 3.0(n=6)$, for a black hole 
mass $M=5 \mathrm{TeV} ; w / w_{\text {typical }} \approx 3.6(n=2), w / w_{\text {typical }} \approx$ $3.3(n=4)$ and $w / w_{\text {typical }} \approx 3.1(n=6)$, for $M=10$ TeV. $w_{\text {typical }}$ varies in the interval $\sim 100-165 \mathrm{GeV}$, depending on $n$ and $M$. The contribution of distances shorter than the new Planck length $l_{T e V} \sim 10^{-17} \mathrm{~cm}$ to the spectrum reaches now significative values: $21 \%$ $(n=2), 25 \%(n=4)$ and $28 \%(n=6)$ for $M=5$ $\mathrm{TeV}$, and $17 \%(n=2), 22 \%(n=4)$ and $26 \%(n=6)$ for $M=10 \mathrm{TeV}$ (see Fig. 1). In addition, the relative contribution to the luminosity, originated in the distance range $\left|u_{2}-u_{1}\right|<l_{T e V}$, increases these numbers since grey-body factors $\Gamma_{l}(w)$ grow up with frequency. Since in the ultrashort distance regime there may exist some unknown physics, not described by relativistic quantum field theory, it can give some signature in the evaporation, even before reaching the Planck-scale phase. In other words, significant deviations from the Planckian spectrum can potentially emerge in the "Schwarzschild phase" of the evaporation, where most of the energy is expected to be radiated away [6].

Finally we wish to stress that Eq. (10) can be rewritten as an integral along $I^{-}$(with respect to $d v_{1} d v_{2}$ ). Constraining distances also at $I^{-}$in the "naive" way: $\left(v_{2}-v_{1}\right)^{2} \sim \kappa^{-2}\left(e^{-\kappa u_{2}}-e^{-\kappa u_{2}}\right)^{2}<\epsilon$ is problematic. To see this let us consider Minkowski space and the transformation $v=e^{-\xi} u$, which can be regarded as a radial boost with rapidity $\xi$. Absence of particle production under this boost requires that, at $I^{-}$, we should impose $\left(v_{2}-v_{1}\right)^{2}<\epsilon^{2} e^{-2 \xi}$ (if $\left(u_{2}-u_{1}\right)^{2}<\epsilon^{2}$ ) or $\left(u_{2}-u_{1}\right)^{2}<\epsilon^{2} e^{2 \xi}$ (if $\left.\left(v_{2}-v_{1}\right)^{2}<\epsilon^{2}\right)$. Therefore, under a general transformation $v=v(u$ ) (as the one $v \sim \kappa^{-1} e^{-\kappa u}$ appearing in black hole formation) we should generalize the above relations and the easiest way is $\left(v_{2}-v_{1}\right)^{2}<\epsilon^{2} \frac{d v_{1}}{d u_{1}} \frac{d v_{2}}{d u_{2}}\left(\right.$ if $\left.\left(u_{2}-u_{1}\right)^{2}<\epsilon^{2}\right)$ or $\left(u_{2}-u_{1}\right)^{2}<\epsilon^{2} \frac{d u_{1}}{d v_{1}} \frac{d u_{2}}{d v_{2}}$ (if $\left.\left(v_{2}-v_{1}\right)^{2}<\epsilon^{2}\right)$. In the former situation (naturally preferred since physical measurements are performed at $I^{+}$) the results are equivalent to those obtained previously and parallel to those obtained in sonic black holes. The second possibility is more exotic since it predicts a drastic change in the particle production rate [21]. The radiation is approximately thermal after the formation of the black hole, but for a short period. Moreover, the correlations cease to be zero and increase with time. This possibility cannot be excluded completely (see also [22]).

We thank A. Fabbri and L. Parker for useful comments and suggestions. I.A. thanks MEC for a FPU fellowship. This work has been partially supported by grants FIS2005-05736-C03-03 and EU network MRTNCT-2004-005104. G.J.O. has been supported by NSF grants PHY-0071044 and PHY-0503366.
[1] S. W. Hawking, Comm. Math. Phys. 43199 (1975)

[2] G.'t Hooft, Nucl. Phys. B335, 138 (1990)

[3] T. Jacobson, Phys. Rev. D 441731 (1991); Phys. Rev D 48728 (1993)

[4] W.G. Unruh, Phys. Rev. D 512827 (1995)

[5] R. Brout, S. Massar, R. Parentani and P. Spindel, Phys. Rev. D 524559 (1995); S. Corley and T. Jacobson, Phys. Rev. D 541568 (1996); Phys. Rev. D 59124011 (1999); S. Corley, Phys. Rev. D 576280 (1998)

[6] S.B. Giddings and S. Thomas, Phys. Rev. D 65, 056010 (2002). S.B. Giddings, Gen.Rel.Grav. 34, 1775 (2002). B.J. Carr and S.B. Giddings, Sci.Am. 292 N5:30-37 (2005)

[7] S. Dimopoulos and G. Landsberg, Phys. Rev. Lett. 87, 161602 (2001).

[8] S. Dimopoulos and R. Emparan, Phys. Lett. B 526, 393 (2002). G. Landsberg, Phys. Rev. Lett. 88181801 (2002). C.M. Harris and P. Kanti, JHEP 0310:014 (2003). J.L. Hewett, B. Lillie and T. Rizzo, Phys. Rev. Lett. 95 261603 (2005)

[9] N. Arkani-Hamed, S. Dimopoulos and G.R. Dvali, Phys. Lett. B 429, 263 (1998). I. Antoniadis, N. Arkani-Hamed, S. Dimopoulos and G.R. Dvali, Phys. Lett. B 436, 257 (1998). L. Randall and R. Sundrum, Phys. Rev. Lett. 83,
3370 (1999)

[10] T. Banks and W. Fischler, hep-th/9906038

[11] R. Emparan, G.T. Horowitz and R.C. Myers, Phys. Rev. Lett. 85499 (2000)

[12] L. Parker Phys. Rev. D 12, 1519 (1975)

[13] R. M. Wald Commun. Math. Phys. 45, 9 (1975)

[14] R. M. Wald Quantum field theory in curved spacetime and black hole thermodynamics, CUP, Chicago (1994)

[15] A. Fabbri and J. Navarro-Salas Modeling black hole evaporation, ICP-World Scientific, London (2005)

[16] A. Fabbri, J. Navarro-Salas and G.J. Olmo, Phys. Rev. D 70064022 (2004)

[17] K. Fredenhagen and R. Haag, Commun. Math. Phys. 127 273 (1990)

[18] G.J. Olmo, Ph.D Thesis, University of Valencia (2005)

[19] C. Barceló, S. Liberati and M. Visser, Living Rev. Rel. 8:12 (2005); R. Balbinot, A. Fabbri, S. Fagnocchi and R. Parentani, Riv. Nuovo Cimento 28, 1 (2005) gr-qc/0601079

[20] R.C.Myers and M.J.Perry,Ann.Phys.(N.Y.)172,304(1986)

[21] Work in preparation

[22] W.G. Unruh and R. Schutzhold, Phys. Rev. D 71, 024028 (2005) 